\title{
Duodenoscope-associated infection prevention: A call for evidence-based decision making
}

\section{다(1) $(9$}

\author{
Authors \\ Institutions \\ 1 Ofstead \& Associates, Inc., St. Paul, Minnesota, United \\ States \\ 2 Boston University Medical Center, Boston, \\ Massachusetts, United States
}

Cori L. Ofstead ${ }^{1}$, Brandy L. Buro' ${ }^{1}$, Krystina M. Hopkins ${ }^{1}$, John E. Eiland ${ }^{1}$, Harry P. Wetzler ${ }^{1}$, David R. Lichtenstein ${ }^{2}$

submitted 21.5 .2020

accepted after revision 30.7 .2020

Bibliography

Endoscopy International Open 2020; 08: E1769-E1781

DOI 10.1055/a-1264-7173

ISSN 2364-3722

(c) 2020. The Author(s).

This is an open access article published by Thieme under the terms of the Creative Commons Attribution-NonDerivative-NonCommercial License, permitting copying and reproduction so long as the original work is given appropriate credit. Contents may not be used for commecial purposes, or adapted, remixed, transformed or built upon. (https://creativecommons.org/licenses/by-nc-nd/4.0/)

Corresponding author

Cori L. Ofstead, MSPH, President and CEO, Ofstead \&

Associates, Inc., 1360 Energy Park Drive, Suite 300, Saint Paul, MN 55108, United States

Fax: +1-651-647-6287

cori@ofsteadinsights.com

\section{ABSTRACT}

Background Recent outbreaks of duodenoscope-associated multidrug-resistant organisms (MDROs) have brought attention to the infection risk from procedures performed with duodenoscopes. Prior to these MDRO outbreaks, pro- cedures with duodenoscopes were considered safe and low risk for exogenous infection transmission, provided they were performed in strict accordance with manufacturer instructions for use and multisociety reprocessing guidelines. The attention and efforts of the scientific community, regulatory agencies, and the device industry have deepened our understanding of factors responsible for suboptimal outcomes. These include instrument design, reprocessing practices, and surveillance strategies for detecting patient and instrument colonization. Various investigations have made it clear that current reprocessing methods fail to consistently deliver a pathogen-free instrument. The magnitude of infection transmission has been underreported due to several factors. These include the types of organisms responsible for infection, clinical signs presenting in sites distant from ERCP inoculation, and long latency from the time of acquisition to infection. Healthcare providers remain hampered by the ill-defined infectious risk innate to the current instrument design, contradictory information and guidance, and limited evidence-based interventions or reprocessing modifications that reduce risk. Therefore, the objectives of this narrative review included identifying outbreaks described in the peer-reviewed literature and comparing the findings with infections reported elsewhere. Search strategies included accessing peer-reviewed articles, governmental databases, abstracts for scientific conferences, and media reports describing outbreaks. This review summarizes current knowledge, highlights gaps in traditional sources of evidence, and explores opportunities to improve our understanding of actual risk and evidencebased approaches to mitigate risk.

\section{Evolution of endoscopy-associated infection risk estimates}

\section{Original risk estimates}

For many years, clinicians and policymakers believed endoscopy-associated infections were extremely rare. This belief was bolstered when a 1993 position paper by the American Society for Gastrointestinal Endoscopy estimated that infections associated with gastrointestinal endoscopy occurred in 1 in 1.8 mil- lion procedures [1] and other guidelines repeated this risk estimate [2-5].

Infection attack rates are calculated by identifying all exposed patients (denominator) and actively assessing them to determine the number infected or colonized (numerator) ( Fig.1). However, the oft-repeated infection risk estimate was calculated using a numerator of 28 upper and lower gastrointestinal endoscopy cases, including endoscopic retrograde cholangiopancreatography (ERCP), derived from a 1993 literature review by Spach et al. [6]. and a denominator of 40 million, 
Number of exposed patients who became infected or colonized

Total number of exposed patients

- Fig. 1 Infection attack rate equation.

which was a "guesstimate" of the United States procedural volume in 1988 to 1992 [1]. Spach et al. cautioned against using their data to calculate infection risk and stated, "These recognized and reported cases, however, probably represent a minority of all infections transmitted by endoscopy, because they were primarily due to easily recognized bacterial infections characterized by short incubation periods and often occurring in large or unusual clusters." [6]. They concluded "Given the above limitations and lack of prospective studies, the true incidence of infections transmitted by endoscopy is impossible to determine" and recommended that prospective studies include monitoring patients for clinical disease and positive cultures following endoscopy [6].

Outbreaks following ERCP are often detected only because multidrug-resistant organisms (MDRO) attract the attention of clinicians and infection preventionists. Recognition of other infections may be limited by pitfalls that adversely impact detection, including the following issues that are described in this article:

- A failure to detect asymptomatic colonization due to a lack of routine post-ERCP screening cultures;

- Long lag times between procedures and the appearance of clinical infections;

- Remote infection sites in the body that are not recognized as ERCP-related; and

- The transmission of bacteria that are typically endogenous gut flora (assumed to have originated in the infected patient).

Although the inaccuracy of endoscopy-associated infection risk estimates was described in 2013 [7], the risk continues to be characterized as less than one in a million or "extremely rare" [8-10] without any substantiating evidence. The concept of negligible risk has been used to support clinical decision making (e.g., declining to notify or test exposed patients), even when serious reprocessing breaches were identified [11-13]. Additionally, incomplete risk estimates for endoscopic procedures adversely impact the informed consent process and can leave patients with a false sense of security. Thus, there remains a need to prospectively monitor large numbers of endoscopy patients to accurately determine attack rates.

Ill-defined infection risk estimates may jeopardize patient safety. Therefore, the objectives of this narrative review include identifying outbreaks and reprocessing failures described in peer-reviewed literature and comparing the findings with evidence reported elsewhere. Traditional search strategies were used to identify articles indexed in PubMed that described infections, reprocessing breaches, and residual contamination on duodenoscopes. In addition, researchers reviewed evidence of infections and reprocessing failures described in governmental databases and reports, abstracts for scientific conferences, and media reports describing outbreaks.

\section{Risks based on retrospective analyses}

Recent retrospective studies have documented higher rates of post-endoscopy infection, which provide a starkly different view of infection risk. Additionally, advances in genetic testing and molecular technology now allow investigators to detect outbreak organisms and link them directly to contaminated endoscopes [14].

Responding to concerns about historic infection risk estimate accuracy [15] and a safety communication from the US Food and Drug Administration (FDA) regarding pathogen transmission associated with ERCP [16], Wang et al. used claims data to determine the risk of endoscopy-associated infections requiring emergency care or hospitalization within seven days of procedures [17]. The researchers hypothesized that facilityrelated factors may contribute to post-endoscopy infections. They reviewed records for 2,347,894 colonoscopy, gastroscopy, bronchoscopy, and cystoscopy procedures in ambulatory surgery centers (ASCs) in six states [17]. The overall infection risk varied by procedure type and was far higher than previously asserted $(1.1,3.0$, and 15.6 per 1,000 for screening colonoscopy, esophagogastroduodenoscopy, and bronchoscopy, respectively) [17]. The risk differed by setting, with serious infections transmitted to more than $10 \%$ of patients in certain ASCs [17]. This demonstrated that endoscopy-associated infections were not "very rare" (defined by the World Health Organization [WHO] as $<1 / 10,000$ patients) or even "rare" $(<1 / 1,000$ patients) [18], even for gastrointestinal endoscopes lacking elevator mechanisms.

There is a paucity of data from large multisite studies on infection risk for patients who have undergone ERCP. Loor et al. analyzed surveillance data to determine the impact of preoperative ERCP on cholecystectomy surgical site infection (SSI) risk [19]. Patients undergoing pre-operative ERCP had more than double the SSI rate ( $4.1 \%$ vs $1.8 \%$ ), with more resistant pathogens ( $1.1 \%$ vs $0.2 \%$ ) compared to those without pre-operative ERCP [19]. This suggests pathogen transmission during ERCP may remain undetected until later invasive procedures. The study was conducted in an institution that had previously found $60 \%$ of patient-ready endoscopes harbored bacteria (including gram-negative organisms linked to contaminated AER rinse water) despite adherence to reprocessing guidelines [20]. Researchers from that hospital subsequently described the transmission of an MDRO from a patient with a pre-existing infection to a gastroscope that harbored the pathogen through 12 reprocessing cycles and procedures involving nine other patients [21]. These studies established that repeated cycles of reprocessing in that facility did not remove potential pathogens from endoscopes, and thus it is possible that the postERCP SSIs reported by Loor et al. could have been transmitted by contaminated duodenoscopes. 


\section{Risk based on outbreaks reported in peer-reviewed journals}

When attack rates were calculated using data from 15 duodenoscope-associated outbreaks, the lowest rate was $6 \%$, and attack rates were $\geq 20 \%$ in nine outbreaks ( $\vee$ Table 1 ). Only three manuscripts explicitly reported attack rates, which ranged from $14 \%$ to $41 \%$ [22-24]. In five manuscripts, investigators documented secondary transmission to close contacts of ERCP patients and included these in the total number of infections. Sometimes the number of exposed patients was not reported, or exposed patients were not notified or tested ( $\triangleright$ Table 1 ). Rectal culture sensitivity is uncertain and may underestimate transmission from a contaminated endoscope. Together, these factors limit the ability to calculate accurate attack rates.

Peer-reviewed literature is often considered to be the definitive source of scientific evidence. However, the information about duodenoscope-associated outbreaks in journals was often incomplete or contradicted by evidence found elsewhere, such as FDA adverse event (AE) (MAUDE) reports, Centers for Disease Control and Prevention (CDC) investigations, and health department inspections. T Table 2 provides additional details about four outbreaks described in peer-reviewed articles included in $>$ Table 1 . Different sources of information often reported contradictory numbers of affected patients and infection rates for the same outbreak (e. g., Illinois: $26.5 \%$ [25] to $46.0 \%$ [26]). There was poor alignment regarding other aspects of the outbreaks, including the number of duodenoscopes involved, pathogens found, and the presence of reprocessing breaches ( $>$ Table 2 ).

\section{Infections reported to federal and state agencies}

In 2017-2019, numerous reports of breaches and infections attributed to contaminated duodenoscopes were submitted to the FDA ( $\downarrow$ Table 3 ). MAUDE reports often provide information about microbial culture results, device damage or malfunctions, affected patients, reprocessing breaches, and endoscope maintenance issues. For example, in 2019, a series of reports described 32 ERCP patients infected with vancomycin- and carbapenem-resistant organisms in one institution since 2013, including 12 cases and one death in 2018. Serious reprocessing breaches were identified by the manufacturer, including problems with point-of-care precleaning, delayed reprocessing, manual cleaning, irrigation systems, handling, and transport $[27,28]$. Despite these reported breaches, the reports indicated the state health department investigated and observed "no abnormalities" [27]. This outbreak has not been published in peer-reviewed literature or news media, nor has it been factored into infection risk estimates and trends. This example and our review of infections reported to federal and state agencies strongly suggest that infections are underreported.

\section{Root cause of duodenoscope-associated infections}

\section{Pathogen, procedural, and patient risk factors}

ERCP-related infections develop as a result of a complex interplay between bacterial pathogens, procedural factors, and underlying pancreaticobiliary (PB) anatomy. Duodenoscopes are exposed to normal flora and potential pathogens during passage through the oral cavity, esophagus, and duodenum, and the risk of endogenous transmission has long been recognized $[3,14]$. These bacteria, which are endogenous, can be introduced into the PB tree during ERCP, leading to a spectrum of infectious complications ranging from transient bacteremia to cholecystitis, cholangitis, and infected pancreatic fluid collections (e.g., pseudocyst, walled-off pancreatic necrosis or cystic neoplasm) [29]. Transient bacteremia occurs in up to $15 \%$ of diagnostic and $28 \%$ of therapeutic ERCP procedures, but infrequently progresses to sepsis among immunocompetent patients [30-33]. Although antibiotic prophylaxis has been shown to reduce the incidence of bacteremia associated with ERCP, pre-procedure antibiotic prophylaxis has not been shown to prevent cholangitis [31,32].

PB infectious complications typically occur as a result of instrumentation or contrast injection into an incompletely drained PB tree [29]. Other risk factors may include underlying immunocompromised state. Bacterial contaminants already present on a "patient-ready" duodenoscope - termed exogenous pathogens - can produce a similar range of infectious PB complications as well as intestinal colonization that can persist or lead to remote sites of infection in the urinary tract, pulmonary tree or bloodstream up to months after the initial ERCP [25].

\section{Exogenous flora and reprocessing effectiveness}

Given the exposure of reusable duodenoscopes to blood, gastric secretions, enteric microbiota, and potential pathogens, effective reprocessing is essential to remove soil and bioburden and prevent the transmission of exogenous pathogens, including MDROs. Although manual cleaning and high-level disinfection (HLD) should theoretically eliminate all microbes except resilient bacterial spores, recent studies have demonstrated that duodenoscope reprocessing is not reliably effective ( $\triangleright$ Table 4 ) [34-38].

Reprocessing failures may occur in part due to the complex distal end of duodenoscopes, which have elevator mechanisms. The elevator, open wires, and channels are exposed to bioburden during procedures, and the instrument design of conventional models does not allow disassembly or direct visualization during cleaning. Numerous infections have been attributed to pathogens detected on elevator mechanisms, wires, or channels [39-42]. However, outbreak strains have also been detected in other duodenoscope components including the suctionbiopsy channel [22,42-44].

In light of these reprocessing failures and outbreaks linked to contaminated duodenoscopes, the FDA recommended in 2015 that institutions adopt enhanced methods of reprocessing, such as double HLD or sterilization [45]. Since then, re- 
- Table 1 Duodenoscope-associated infections reported in peer-reviewed journal articles.

\begin{tabular}{|c|c|c|c|c|c|c|c|c|}
\hline \multirow[t]{2}{*}{ Source $^{1}$} & \multirow[t]{2}{*}{ Location } & \multirow{2}{*}{$\begin{array}{l}\text { Pathogens isolated } \\
\text { from patients }\end{array}$} & \multirow{2}{*}{$\begin{array}{l}\text { Infec- } \\
\text { ted } \\
\text { pa- } \\
\text { tients }\end{array}$} & \multirow{2}{*}{$\begin{array}{l}\text { Ex- } \\
\text { posed } \\
\text { to } \\
\text { scopes }\end{array}$} & \multirow{2}{*}{$\begin{array}{l}\text { Post-expo- } \\
\text { sure test- } \\
\text { ing }\end{array}$} & \multirow{2}{*}{$\begin{array}{l}\text { Reported } \\
\text { attack rate }\end{array}$} & \multicolumn{2}{|c|}{ Calculated attack rate } \\
\hline & & & & & & & Rate $^{2}$ & $\begin{array}{l}\text { Confi- } \\
\text { dence }^{3}\end{array}$ \\
\hline $\begin{array}{l}\text { Rauwers } \\
2019[43]\end{array}$ & Utrecht, Netherlands & $\begin{array}{l}\text { MDR Klebsiella pneu- } \\
\text { moniae }\end{array}$ & $27^{4}$ & 102 & $81(79.4 \%)$ & $\begin{array}{l}35 \% \text { scope A } \\
29 \% \text { scope B }\end{array}$ & $\begin{array}{l}32.5 \% \\
{[26 / 80]}\end{array}$ & Medium \\
\hline $\begin{array}{l}\text { Bourigault } \\
2018 \text { [97] }\end{array}$ & Nantes, France & $\begin{array}{l}\text { CR K. pneumoniae } \\
\text { (OXA-48) }\end{array}$ & 5 & 61 & $41(67.2 \%)$ & NR & $\begin{array}{l}12.2 \% \\
{[5 / 41]}\end{array}$ & Medium \\
\hline $\begin{array}{l}\text { Shenoy } \\
2018[98]\end{array}$ & Boston, MA, USA & mcr-1 K. pneumoniae & $1^{5}$ & 5 & $5(100 \%)$ & NR & $20 \%[1 / 5]$ & High \\
\hline $\begin{array}{l}\text { Robertson } \\
2017 \text { [99] }\end{array}$ & Glasgow, Scotland & Salmonella enteritidis & $4^{6}$ & 9 & $9(100 \%)$ & NR & $37.5 \%[3 / 8]$ & High \\
\hline $\begin{array}{l}\text { Kim } \\
2016[23]\end{array}$ & Los Angeles, CA, USA & $\begin{array}{l}\text { CR K. pneumoniae } \\
\left(\text { bla } a_{\text {OXA-232 }}\right)\end{array}$ & 15 & 115 & $104(90.4 \%)$ & $14.4 \%$ & $\begin{array}{l}14.4 \% \\
{[15 / 104]}\end{array}$ & Medium \\
\hline $\begin{array}{l}\text { Kola } \\
2015 \text { [100] }\end{array}$ & Berlin, Germany & $\begin{array}{l}\text { CR K. pneumoniae } \\
\text { (OXA-48) }\end{array}$ & $\begin{array}{l}12[6 \\
\text { ERCP }]\end{array}$ & 26 & $23(88.5 \%)$ & NR & $\begin{array}{l}26.1 \% \\
{[6 / 23]}\end{array}$ & Medium \\
\hline $\begin{array}{l}\text { Marsh } \\
2015 \text { [101] }\end{array}$ & Pittsburgh, PA, USA & $\begin{array}{l}\text { CR K. pneumoniae } \\
\text { ESBL K. pneumoniae }\end{array}$ & $\begin{array}{l}34[12 \\
\text { ERCP }]\end{array}$ & UNK & UNK & NR & - & - \\
\hline $\begin{array}{l}\text { Wendorf } \\
2015[96]\end{array}$ & Seattle, WA, USA & $\begin{array}{l}\text { AmpC E. coli } \\
\text { CR E. coli }\end{array}$ & 35 & UNK & 49 & NR & - & - \\
\hline $\begin{array}{l}\text { Verfaillie } \\
2015 \text { [39] }\end{array}$ & $\begin{array}{l}\text { Rotterdam, Nether- } \\
\text { lands }\end{array}$ & VIM-2 P. aeruginosa & $\begin{array}{l}30[22 \\
\text { ERCP }]\end{array}$ & 251 & UNK & NR & - & - \\
\hline $\begin{array}{l}\text { Qiu } \\
2015 \text { [102] }\end{array}$ & Hangzhou, China & P. aeruginosa & 3 & 3 & $3(100 \%)$ & NR & $100 \%[3 / 3]$ & High \\
\hline $\begin{array}{l}\text { Smith } \\
2015[72]\end{array}$ & Milwaukee, WI, USA & NDM-1 E. coli & $4^{7}$ & 27 & $18(66.7 \%)$ & NR & $\begin{array}{l}23.5 \% \\
{[4 / 17]}\end{array}$ & Medium \\
\hline $\begin{array}{l}\text { Epstein } \\
2014[25]\end{array}$ & Chicago, IL, USA & NDM, CR E. coli & $\begin{array}{l}39[35 \\
\text { ERCP }]\end{array}$ & 226 & $102(45.1 \%)$ & NR & $\begin{array}{l}26.5 \% \\
{[27 / 102]}\end{array}$ & Low \\
\hline $\begin{array}{l}\text { Alrabaa } \\
2013[40]\end{array}$ & Tampa, FL, USA & CR K. pneumoniae & 10 & 51 & $46(90.2 \%)$ & NR & $\begin{array}{l}21.7 \% \\
{[10 / 46]}\end{array}$ & Medium \\
\hline $\begin{array}{l}\text { Carbonne } \\
2010[22]\end{array}$ & Paris, France & $\begin{array}{l}\text { CR K. pneumoniae } \\
\text { (KPC-2) }\end{array}$ & $\begin{array}{l}12[7 \\
\text { ERCP }]^{5}\end{array}$ & 17 & $16(94.1 \%)$ & $41 \%$ & $\begin{array}{l}43.8 \% \\
{[7 / 16]}\end{array}$ & Medium \\
\hline $\begin{array}{l}\text { Aumeran } \\
2010[44]\end{array}$ & $\begin{array}{l}\text { Clermont-Ferrand, } \\
\text { France }\end{array}$ & ESBL K. pneumoniae & 16 & 253 & $253(100 \%)$ & NR & $\begin{array}{l}6.3 \% \\
{[16 / 253]}\end{array}$ & High \\
\hline
\end{tabular}

MDR, multidrug resistant; NR, not reported in source article; CR, carbapenem-resistant; NDM: New Delhi beta-lactamase producing; mcr-1, Mobile colistin resistance gene 1; AmpC, cefoxitin/third-generation cephalosporin resistant, carbapenem sensitive; ESBL, extended-spectrum beta-lactamase producing; VIM-2, Verona integron-borne metallo-beta-lactamase producing

${ }^{1}$ When an outbreak has multiple published sources, only the first publication was included in this table.

${ }^{2}$ Attack rates were calculated by dividing the number of outbreak patients with duodenoscope exposure by the number of patients with duodenoscope exposure

who were subsequently tested. Patients identified by investigators as index or source patients were removed from the numerator and denominator for accuracy.

${ }^{3}$ Confidence ranked as follows: High: $100 \%$ of exposed patients were tested; Medium: $\geq 66 \%$ of exposed patients were tested; Low: <66\% of exposed patients were

tested.

${ }^{4}$ Number of infected patients includes an index patient identified by investigators.

${ }^{5}$ Investigators clearly identified the source patient as the individual who introduced the pathogen into the scope; we excluded the source patient from the number of patients infected during the outbreak.

${ }^{6}$ Investigators hypothesized-but did not confirm-that the index patient was also the source patient.

${ }^{7}$ Investigators identified a source patient but included this patient in the number of patients exposed and tested; we excluded the source patient from the number of patients infected and from the denominator in the attack rate calculation.

searchers have determined that double HLD is no more effective than single HLD [34-38], with double-HLD failure rates ranging from $2 \%$ [38] to $44 \%$ [37] ( $\triangleright$ Table 4). Although sterilization should theoretically be failproof, several researchers have reported microbial growth in samples from duodenoscopes following sterilization with ethylene oxide (23\% [36],
$18 \%$ [46]) and peracetic acid (2\%) [38] and from ureteroscopes following hydrogen peroxide gas sterilization (13\%) [47].

Similar reprocessing failures have been found with curvilinear array echoendoscopes (EUS), which also have an elevator mechanism. Chapman et al. performed 540 microbial cultures on 18 patient-ready EUS endoscopes and found $4.2 \%$ were po- 
Table 2 Comparison of evidence from multiple sources describing four duodenoscope-associated outbreaks.

\begin{tabular}{|c|c|c|c|c|c|c|c|c|}
\hline $\begin{array}{l}\text { Location } \\
\text { [Pathogen] }\end{array}$ & Source & $\begin{array}{l}\text { Source } \\
\text { type }\end{array}$ & $\begin{array}{l}\text { Case } \\
\text { patients }^{1}\end{array}$ & $\begin{array}{l}\text { Ex- } \\
\text { posed }\end{array}$ & Tested & $\begin{array}{l}\text { Posi- } \\
\text { tive } \\
\text { test }\end{array}$ & $\begin{array}{l}\text { Attack rate } \\
\text { (Positive/ } \\
\text { Tested) }\end{array}$ & Comments \\
\hline \multirow[t]{3}{*}{$\begin{array}{l}\text { Tampa Gen- } \\
\text { eral Hospital, } \\
\text { Tampa, FL } \\
\text { [CR K. pneu- } \\
\text { moniae] }\end{array}$} & $\begin{array}{l}\text { Alrabaa } 2013 \\
{[40]}\end{array}$ & $\begin{array}{l}\text { Journal ar- } \\
\text { ticle }\end{array}$ & 10 & 51 & 46 & 10 & $\begin{array}{l}21.7 \%{ }^{2} \\
{[10 / 46]}\end{array}$ & $\begin{array}{l}\text { - Reprocessing breaches re- } \\
\text { ported } \\
\text { - Bio-debris visible under ele- } \\
\text { vator }\end{array}$ \\
\hline & $\begin{array}{l}\text { Sanderson } \\
2010[103]\end{array}$ & $\begin{array}{l}\text { APIC ab- } \\
\text { stract }\end{array}$ & $\begin{array}{l}16[9 \\
E R C P]\end{array}$ & 51 & 46 & 9 & $\begin{array}{l}19.6 \%^{2} \\
{[9 / 46]}\end{array}$ & $\begin{array}{l}\text { - Endoscope contamination } \\
\text { (Escherichia coli, Pseudomonas, } \\
\text { Serratia spp.) }\end{array}$ \\
\hline & $\begin{array}{l}\text { Sanderson } \\
2010[104]\end{array}$ & $\begin{array}{l}\text { APIC pre- } \\
\text { sentation }\end{array}$ & $\begin{array}{l}14 \text { total } \\
\text { Site A: } 7 \\
\text { Site } B: 7\end{array}$ & $\begin{array}{l}\text { Site A: } \\
51 \\
\text { Site B: } \\
140\end{array}$ & $\begin{array}{l}\text { Site A: } \\
22 \\
\text { Site B: } \\
140\end{array}$ & $\begin{array}{l}\text { Site A: } \\
7 \\
\text { Site B: } \\
7\end{array}$ & $\begin{array}{l}\text { Site A: } \\
31.8 \%^{2} \\
{[7 / 22]} \\
\text { Site B: } 5 \% \\
{[7 / 140]}\end{array}$ & $\begin{array}{l}\text { - Endoscope contamination } \\
\text { (Pseudomonas aeruginosa, } \\
\text { Proteus mirabilis, and E. coli) } \\
\text { - Secondary transmission to } \\
\text { other hospitals was found }\end{array}$ \\
\hline \multirow{5}{*}{$\begin{array}{l}\text { Advocate } \\
\text { Lutheran } \\
\text { General Hos- } \\
\text { pital, Chicago, } \\
\text { IL [NDM-pro- } \\
\text { ducing CR E. } \\
\text { coli] }\end{array}$} & $\begin{array}{l}\text { Epstein } 2014 \\
\text { [25] }\end{array}$ & $\begin{array}{l}\text { Journal ar- } \\
\text { ticle }\end{array}$ & $\begin{array}{l}39[35 \\
\text { ERCP] }\end{array}$ & 226 & 102 & 27 & $\begin{array}{l}26.5 \%^{2} \\
{[27 / 102]}\end{array}$ & $\begin{array}{l}\text { - No reprocessing breaches } \\
\text { reported, but IFU deviations } \\
\text { are described }\end{array}$ \\
\hline & Ray 2018 [90] & $\begin{array}{l}\text { Journal ar- } \\
\text { ticle }\end{array}$ & 31 & UNK & UNK & UNK & - & $\begin{array}{l}\text { - Secondary transmission to } 10 \\
\text { patients at } 6 \text { other hospitals }\end{array}$ \\
\hline & Frias 2014 [26] & $\begin{array}{l}\text { CDC } \\
\text { MMWR }\end{array}$ & 44 & 91 & 50 & 23 & $\begin{array}{l}46.0 \% \\
{[23 / 50]}\end{array}$ & $\begin{array}{l}\text { - No reprocessing breaches } \\
\text { reported }\end{array}$ \\
\hline & $\begin{array}{l}\text { Epstein } 2013 \\
\text { [41] }\end{array}$ & $\begin{array}{l}\text { CDC Epi- } \\
\text { Aid } \\
\text { Trip Report }\end{array}$ & $\begin{array}{l}26[23 \\
\text { ERCP }]\end{array}$ & 96 & 45 & 17 & $\begin{array}{l}37.8 \% \\
{[17 / 45]}\end{array}$ & $\begin{array}{l}\text { - Endoscope damage reported } \\
\text { - Inadequate hand hygiene and } \\
\text { PPE }\end{array}$ \\
\hline & $\begin{array}{l}\text { CMS } 2014 \\
{[105]}\end{array}$ & $\begin{array}{l}\text { Statement } \\
\text { of Defi- } \\
\text { ciencies }\end{array}$ & 38 & 243 & 114 & 38 & $\begin{array}{l}33.3 \%^{2} \\
{[38 / 114]}\end{array}$ & $\begin{array}{l}\text { - Reprocessing breaches re- } \\
\text { ported }\end{array}$ \\
\hline \multirow{6}{*}{$\begin{array}{l}\text { UCLA Medical } \\
\text { Center, Los } \\
\text { Angeles, CA } \\
\text { [CR K. pneu- } \\
\text { moniae] }\end{array}$} & Kim 2016 [23] & $\begin{array}{l}\text { Journal ar- } \\
\text { ticle }\end{array}$ & 15 & 115 & 104 & 15 & $\begin{array}{l}14.4 \% \\
{[15 / 104]}\end{array}$ & $\begin{array}{l}\text { - No reprocessing breaches } \\
\text { reported }\end{array}$ \\
\hline & $\begin{array}{l}\text { Humphries } \\
2017[73]\end{array}$ & $\begin{array}{l}\text { Journal ar- } \\
\text { ticle }\end{array}$ & $16^{3}$ & 179 & 150 & 8 & $\begin{array}{l}5.3 \%^{2} \\
{[8 / 150]}\end{array}$ & $\begin{array}{l}\text { - No reprocessing breaches } \\
\text { reported }\end{array}$ \\
\hline & $\begin{array}{l}\text { Yang } 2018 \\
{[106]}\end{array}$ & $\begin{array}{l}\text { Journal ar- } \\
\text { ticle }\end{array}$ & $16^{3}$ & UNK & UNK & UNK & - & $\begin{array}{l}\text { - Endoscopes and reprocessing } \\
\text { practices were not evaluated }\end{array}$ \\
\hline & $\begin{array}{l}\text { UCLA } 2015 \\
{[107]}\end{array}$ & $\begin{array}{l}\text { Public } \\
\text { statement }\end{array}$ & 7 & $>100$ & UNK & UNK & - & $\begin{array}{l}\text { - No reprocessing breaches } \\
\text { reported }\end{array}$ \\
\hline & $\begin{array}{l}\text { Rubin } 2015 \\
{[108]}\end{array}$ & $\begin{array}{l}\text { FDA Panel } \\
\text { presenta- } \\
\text { tion }\end{array}$ & 14 & 179 & 149 & 6 & $\begin{array}{l}4.0 \%^{2} \\
{[6 / 149]}\end{array}$ & $\begin{array}{l}\text { - } 3 \text { deaths reported } \\
\text { - No reprocessing breaches } \\
\text { reported }\end{array}$ \\
\hline & $\begin{array}{l}\text { CMS } 2015 \\
{[109]}\end{array}$ & $\begin{array}{l}\text { Statement } \\
\text { of Defi- } \\
\text { ciencies }\end{array}$ & UNK & UNK & UNK & UNK & - & $\begin{array}{l}\text { - Reprocessing issues are de- } \\
\text { scribed; Immediate Jeopardy } \\
\text { declared } \\
\text { - No environmental cultures } \\
\text { after outbreak }\end{array}$ \\
\hline
\end{tabular}


- Table 2 (Continuation)

\begin{tabular}{|c|c|c|c|c|c|c|c|c|}
\hline $\begin{array}{l}\text { Location } \\
\text { [Pathogen] }\end{array}$ & Source & $\begin{array}{l}\text { Source } \\
\text { type }\end{array}$ & $\begin{array}{l}\text { Case } \\
\text { patients }^{1}\end{array}$ & $\begin{array}{l}\text { Ex- } \\
\text { posed }\end{array}$ & Tested & $\begin{array}{l}\text { Posi- } \\
\text { tive } \\
\text { test }\end{array}$ & $\begin{array}{l}\text { Attack rate } \\
\text { (Positive/ } \\
\text { Tested) }\end{array}$ & Comments \\
\hline \multirow[t]{5}{*}{$\begin{array}{l}\text { Virginia Ma- } \\
\text { son Medical } \\
\text { Center, Seat- } \\
\text { tle, WA [CRE } \\
\text { E. coli and } \\
\text { AmpCE. coli] }\end{array}$} & $\begin{array}{l}\text { Wendorf } 2015 \\
{[96]}\end{array}$ & $\begin{array}{l}\text { Journal ar- } \\
\text { ticle }\end{array}$ & 35 & UNK & 49 & UNK & - & $\begin{array}{l}\text { - No reprocessing breaches } \\
\text { reported } \\
\text { - Endoscope defects (7 of } 8 \\
\text { scopes) } \\
\text { - Endoscope contamination } \\
\text { (AmpC E. coli on } 2 \text { scopes) }\end{array}$ \\
\hline & $\begin{array}{l}\text { Ross } 2015 \\
{[42]}\end{array}$ & $\begin{array}{l}\text { Journal ar- } \\
\text { ticle }\end{array}$ & 32 & 1149 & UNK & UNK & - & $\begin{array}{l}\text { - Endoscope defects ( } 4 \text { of } 8 \\
\text { scopes) } \\
\text { - Endoscope contamination } \\
\text { (AmpC E. coli on } 4 \text { scopes) }\end{array}$ \\
\hline & $\begin{array}{l}\text { Hunter } 2014 \\
{[110]}\end{array}$ & $\begin{array}{l}\text { CDC Epi- } \\
\text { Aid } \\
\text { Trip Report }\end{array}$ & 9 & UNK & UNK & UNK & - & $\begin{array}{l}\text { - No reprocessing breaches } \\
\text { reported, but IFU deviations } \\
\text { described } \\
\text { - Endoscope defects ( } 8 \text { of } 8 \\
\text { scopes) }\end{array}$ \\
\hline & $\begin{array}{l}\text { FDA 2014 } \\
{[111,112]}\end{array}$ & $\begin{array}{l}\text { MAUDE re- } \\
\text { ports }\end{array}$ & 37 & UNK & UNK & UNK & - & - 4 deaths reported \\
\hline & $\begin{array}{l}\text { CMS } 2015 \\
{[113]}\end{array}$ & $\begin{array}{l}\text { Statement } \\
\text { of Defi- } \\
\text { ciencies }\end{array}$ & 39 & 1239 & UNK & UNK & - & $\begin{array}{l}\text { - Reprocessing breaches } \\
\text { reported } \\
\text { - Outbreak detected during } \\
\text { health department study } \\
\text { - Cited for failure to report } \\
\text { outbreak to health depart- } \\
\text { ment }\end{array}$ \\
\hline \multicolumn{9}{|c|}{$\begin{array}{l}\text { CR: carbapenem-resistant; UNK: unknown; -: not reported or not calculated } \\
{ }^{1} \text { Number of case patients included individuals infected or colonized by the outbreak strain and may include patients that were not identified via a formal screening } \\
\text { process. If outbreak investigators reported secondary transmission, the number of patients infected via ERCP is noted in brackets. } \\
{ }^{2} \text { Calculated by Ofstead. } \\
{ }^{3} \text { Outbreak investigators clearly identified the source patient as the individual who introduced the pathogen into the scope; we excluded the source patient from the } \\
\text { number of patients infected during the outbreak. }\end{array}$} \\
\hline
\end{tabular}

sitive for gram-negative organisms [48]. Bartles et al. sampled 45 EUS and ERCP endoscopes 2,925 times and found microbial growth in $7.7 \%$ overall, with growth detected in both the elevator mechanism and the channel [34]. Reprocessing effectiveness studies for other endoscope types reported that microbial growth was found on 35\% [49], $41 \%$ [50], $47 \%$ [51], 58\% [52], $60 \%$ [20, 53], $64 \%$ [54], and $71 \%$ [55] of endoscopes. High-concern organisms (HCOs) were found in most of these studies, which establishes that current reprocessing practices are not reliably effective.

Researchers have identified several factors that impact reprocessing effectiveness, including human factors [52, 56 $59]$, endoscope durability and maintenance issues $[43,47,51$, $53,60-63]$, reprocessing equipment malfunctions $[52,55,64$, $65]$, and difficulty drying endoscopes before storage $[55,61$, $66]$. When HLD or sterilization failed, investigators frequently identified endoscopes with damage or residual soil. In 2010, researchers reported that reprocessing personnel disliked reprocessing tasks, felt pressure to work quickly when reprocessing endoscopes, and experienced physical discomfort from working with endoscopes. These human factors led to reprocessing steps being performed incorrectly or skipped $99 \%$ of the time
[56]. Recent studies have documented widespread nonadherence, with personnel skipping steps or cutting corners due to time pressure and inadequate training and supervision $[35,47$, $52,55,59]$. On the other hand, persistent contamination has been reported even when technicians followed manufacturers' instructions for use (IFU) and guidelines [52 - 54].

\section{Reporting delays}

Major outbreaks have been reported in peer-reviewed literature several years after investigations were initiated [40,43, 67]. In 2014, our team learned of a 2013 ERCP-associated outbreak of New Delhi metallo-beta-lactamase-1 (NDM-1) that occurred in Milwaukee, Wisconsin. No further information was available until a 2015 media article reported that five endoscopy patients had superbug infections [68]. The MAUDE database includes three reports that appear pertinent but were submitted almost a year after the outbreak [69-71]. Clinicians from this institution published a report describing the investigation two years after the outbreak [72].

Reporting delays have also occurred because patients were asymptomatically colonized by pathogens from contaminated 
- Table 3 Infections described in reports submitted to FDA MAUDE database (2017 - 2019).

\begin{tabular}{|c|c|c|c|c|c|}
\hline MAUDE & \# of reports & $\begin{array}{l}\text { Manufac- } \\
\text { turer }\end{array}$ & Infected & Pathogens & $\begin{array}{l}\text { Contributing factors and other } \\
\text { comments }\end{array}$ \\
\hline $\begin{array}{l}8204386[28] \\
8379810[27]\end{array}$ & $33^{1}$ & Olympus & 32 & $\begin{array}{l}\text { VR Enterococcus faecium, CR Enterobac- } \\
\text { teriaceae, Escherichia coli }\end{array}$ & $\begin{array}{l}\text { - } 2 \text { deaths in Texas } \\
\text { - Occurred in } 2016 \text { (20 patients) } \\
\text { and } 2018 \text { - } 2019 \text { ( } 12 \text { patients) } \\
\text { - Reprocessing breach }\end{array}$ \\
\hline $8177954[114]$ & 6 & Olympus & $8[6 \mathrm{ERCP}]$ & MDR P. aeruginosa & $\begin{array}{l}\text { - Cultures were negative for } \\
\text { P. aeruginosa }\end{array}$ \\
\hline $8820754[115]$ & 6 & Olympus & 6 & $\begin{array}{l}\text { CR Enterobacteriaceae, NDM K. pneumo- } \\
\text { niae }\end{array}$ & - Endoscope damage \\
\hline $8538532[116]$ & 6 & Olympus & 5 or 6 & Enterococcus casseliflavus & - Reprocessing breach \\
\hline $7027139[117]$ & 4 & Olympus & 4 & OXA48-producing K. pneumoniae & - Endoscope damage \\
\hline $\begin{array}{l}8201861[118] \\
8201871[119]\end{array}$ & 6 & Olympus & 4 & E. coli, E. faecium (CR + and CR-) & $\begin{array}{l}\text { - Reprocessing breach } \\
\text { - Endoscope contamination }\end{array}$ \\
\hline $7548459[120]$ & 1 & Pentax & $3^{2}$ & MDR P. aeruginosa & \\
\hline $8730284[121]$ & 3 & Olympus & 3 & P. aeruginosa & - Reprocessing breach \\
\hline $8825520[122]$ & 3 & Olympus & 3 & MDR K. pneumoniae & $\begin{array}{l}\text { - Cultures were negative for } \\
\text { K. pneumoniae }\end{array}$ \\
\hline $8751568[123]$ & 1 & Olympus & $1^{2}$ & ESBL K. pneumoniae & - Endoscope contamination \\
\hline $7791919[124]$ & 1 & Olympus & $1^{2}$ & E. casseliflavis & \\
\hline $7424492[125]$ & 1 & Olympus & 1 & MDR Pseudomonas & $\begin{array}{l}\text { - Endoscope contamination } \\
\text { - Endoscope damage }\end{array}$ \\
\hline
\end{tabular}

MDR: Multi-drug resistant; VR: Vancomycin-resistant; NDM: New Delhi metallo-beta-lactamase-producing; ESBL: Extended-spectrum beta-lactamase producing; CR: Carbapenem-resistant/carbapenemase-producing

${ }^{1}$ Reports indicated there are a total of 33 MAUDE reports; we were able to obtain 31 of them.

${ }^{2}$ A source patient (the individual who introduced the pathogen into the endoscope) was clearly identified in the report; we excluded the source patient from the number of patients infected.

duodenoscopes $[22,44]$ and developed clinical signs of infection much later $[23,39,43,73]$. Loor et al. established that SSIs are more common among cholecystectomy patients who had undergone ERCP in the 60 days before surgery [19]. The longterm sequelae of ERCP-associated colonization are not well described. However, a recent report related to superbug colonization following gastroscopy sheds light on the potential impact. Jousset et al. reported that 17 patients were exposed to carbapenemase-producing $K$. pneumoniae during procedures with a contaminated gastroscope [74]. One patient was persistently colonized with the pathogen despite aggressive treatment and experienced fatal sepsis due to this pathogen following prostate and bladder cancer surgery more than 4 years after exposure [74]. This AE was published in 2018, 9 years after the original outbreak. The possibility that patients colonized during ERCP may experience AEs much later should be studied.

\section{FDA recommendations and post-market surveillance studies}

In 2015, the FDA ordered three duodenoscope manufacturers to conduct studies to evaluate the real-world feasibility and effectiveness of reprocessing [75]. Manufacturers found reprocessing staff had difficulty understanding and following in- structions and commonly missed steps [57]. Interim data indicated that HLD failure rates were much higher than the $0.4 \%$ contamination rate anticipated by $\operatorname{FDA}[57,76]$. Final results showed HCOs were present in $4.1 \%$ to $22.2 \%$ of samples, depending on the duodenoscope model, and $0.3 \%$ to $4.4 \%$ had $>$ 100 CFU of low- or moderate-concern organisms. The results released by the FDA represent only a fraction of the number of samples required by the FDA, and hundreds of samples were excluded from analysis for unknown reasons ( $\triangleright$ Table 5) [7680]. The presence of low- and moderate-concern organisms is important because they can contribute to biofilm formation [81]. Although the post-market surveillance studies were designed to demonstrate effectiveness, several MAUDE reports describing microbial growth stated that technicians neglected to follow instructions and made reprocessing errors [82-84]. Despite these findings, FDA maintained that "...an individual's risk of acquiring infection from an inadequately reprocessed medical device remains relatively low given the large number of such devices in use" [76].

The value of these studies is limited by the lack of information about types of institutions submitting data, duodenoscope models, reprocessing methods, personnel adherence, and microbial culture methods. In addition, manufacturers have not reported the proportion of samples with up to 99 CFU of low- 
- Table 4 Effectiveness of HLD, double HLD, and sterilization in real-world settings.

\begin{tabular}{|c|c|c|c|c|c|c|c|c|c|c|}
\hline \multirow[t]{2}{*}{ Study } & \multicolumn{3}{|l|}{ HLD } & \multicolumn{3}{|c|}{ Double HLD } & \multicolumn{3}{|c|}{ Sterilization } & \multirow{2}{*}{$\begin{array}{l}\text { High-concern organ- } \\
\text { isms }\end{array}$} \\
\hline & $\mathbf{N}$ & $\begin{array}{l}\text { Any } \\
\text { growth } \\
(\%)^{1}\end{array}$ & $\begin{array}{l}\text { High- } \\
\text { concern } \\
\text { organ- } \\
\text { isms (\%) }\end{array}$ & $\mathbf{N}$ & $\begin{array}{l}\text { Any } \\
\text { growth } \\
(\%)^{1}\end{array}$ & $\begin{array}{l}\text { High-con- } \\
\text { cern or- } \\
\text { ganisms } \\
(\%)\end{array}$ & $\mathbf{N}$ & $\begin{array}{l}\text { Any } \\
\text { growth } \\
(\%)\end{array}$ & $\begin{array}{l}\text { High-con- } \\
\text { cern or- } \\
\text { ganisms } \\
(\%)\end{array}$ & \\
\hline $\begin{array}{l}\text { Gromski } \\
2019 \\
{[38]}\end{array}$ & - & - & - & 453 & $\begin{array}{l}8 \\
(1.8 \%)\end{array}$ & $2(0.44 \%)$ & $425^{2}$ & $\begin{array}{l}9 \\
(2.1 \%)\end{array}$ & $2(0.47 \%)$ & $\begin{array}{l}\text { Double HLD: Klebsi- } \\
\text { ella pneumoniae; En- } \\
\text { terobacter cloacae } \\
\text { - Sterilization: Strep- } \\
\text { tococcus viridans; } \\
\text { Enterococcus spp. }\end{array}$ \\
\hline $\begin{array}{l}\text { Bartles } \\
2018 \\
{[34]}\end{array}$ & 1399 & $\begin{array}{l}102 \\
(7.3 \%)\end{array}$ & $5(0.4 \%)$ & 1526 & $\begin{array}{l}122 \\
(8.0 \%)\end{array}$ & $3(0.2 \%)$ & - & - & - & $\begin{array}{l}\text { Enterococcus spp.; } \\
\text { Enterobacter cloacae; } \\
\text { Aeromonas spp.; ESBL } \\
\text { +/-Escherichia coli }\end{array}$ \\
\hline $\begin{array}{l}\operatorname{Rex} \\
2017 \\
{[35]}\end{array}$ & - & - & - & $\begin{array}{l}\text { A: } 627 \\
\text { B: } 783^{3}\end{array}$ & $\begin{array}{l}\text { A: } 59 \\
(9.4 \%) \\
\text { B: } 38 \\
(4.9 \%)^{3}\end{array}$ & $\begin{array}{l}\text { A: } 5(0.8 \%) \\
\text { B: } 2(0.3 \%)\end{array}$ & - & - & - & $\begin{array}{l}\text { - Candida glabrata; } \\
\text { Zygomycete; Entero- } \\
\text { coccus spp. }\end{array}$ \\
\hline $\begin{array}{l}\text { Snyder } \\
2017 \\
{[36]}\end{array}$ & 174 & $\begin{array}{l}28 \\
(16.1 \%)\end{array}$ & - & 169 & $\begin{array}{l}27 \\
(16.0 \%)\end{array}$ & - & $173^{4}$ & $\begin{array}{l}39 \\
(22.5 \%)\end{array}$ & - & - Species not reported \\
\hline $\begin{array}{l}\text { Visrodia } \\
2017 \\
{[37]}\end{array}$ & 20 & $\begin{array}{l}12 \\
(60 \%)\end{array}$ & $11(55 \%)$ & 18 & $\begin{array}{l}8 \\
(44.4 \%) \\
5\end{array}$ & - & - & - & - & $\begin{array}{l}\text { Stenotrophomonas } \\
\text { maltophilia; Klebsi- } \\
\text { ella pneumoniae; Pseu- } \\
\text { domonas aeruginosa; } \\
\text { Enterococcus faecalis; } \\
\text { Cellulosimicrobium cel- } \\
\text { lulans }\end{array}$ \\
\hline $\begin{array}{l}\mathrm{N} \text { : number } \\
{ }^{1} \text { Overall g } \\
{ }^{2} \text { Liquid ch } \\
{ }^{3} \mathrm{~A} \text { : Phase } \\
{ }^{4} \text { Ethylene } \\
{ }^{5} \text { Of } 18 \mathrm{scc}\end{array}$ & $\begin{array}{l}\text { encoun } \\
\text { wth rate } \\
\text { ical ster } \\
\text { f study } \\
\text { ide gas s } \\
\text { s that w }\end{array}$ & $\begin{array}{l}\text { rs during w } \\
\text { ported of a } \\
\text { zation usin } \\
\text { en double } \\
\text { rilization in } \\
\text { e re-reproc }\end{array}$ & $\begin{array}{l}\text { ch samples w } \\
\text { microorgan } \\
\text { eracetic acic } \\
\text { D was implen } \\
\text { 3M Sterivac } \\
\text { sed, they onl }\end{array}$ & $\begin{array}{l}\text { e taken for } \\
\text { inc, includir } \\
\text { a Steris } 1 \\
\text { ted. B: } \mathrm{Pr} \\
\text { tem after } \\
\text { ultured } 17\end{array}$ & $\begin{array}{l}\text { crobial cult } \\
\text { igh-concer } \\
\text { stem } \\
\text { III of study } \\
\text { in a Syste }\end{array}$ & $\begin{array}{l}\text { es; -: not evalı } \\
\text { organisms } \\
\text { here new pers } \\
83 \text { Plus } 9 \text { Cust }\end{array}$ & $\begin{array}{l}\text { el wer } \\
\text { Ultras }\end{array}$ & $\begin{array}{l}\text { rained on do } \\
\text { CS AER }\end{array}$ & le HLD & \\
\hline
\end{tabular}

- Table 5 Results from post-market surveillance studies ordered by the FDA in 2015.

\begin{tabular}{|c|c|c|c|c|c|c|c|c|c|c|}
\hline \multirow[t]{2}{*}{$\begin{array}{l}\text { Manu- } \\
\text { facturer }\end{array}$} & \multirow{2}{*}{$\begin{array}{l}\text { Samples } \\
\text { required } \\
\text { by FDA }\end{array}$} & \multicolumn{4}{|c|}{$\begin{array}{l}\text { Interim analysis of all available samples } \\
(2018-2019)\end{array}$} & \multicolumn{4}{|c|}{ Final analysis of properly collected samples (2020) } & \multirow{2}{*}{$\begin{array}{l}\text { Samples } \\
\text { discar- } \\
\text { ded }^{1}\end{array}$} \\
\hline & & $\begin{array}{l}\text { Samples } \\
\text { collected }\end{array}$ & $\begin{array}{l}\text { Ana- } \\
\text { lyzed }\end{array}$ & $\begin{array}{l}\mathrm{HCO} \\
\text { found }\end{array}$ & $\begin{array}{l}\text { High colo- } \\
\text { ny counts }\end{array}$ & $\begin{array}{l}\text { Samples } \\
\text { collected }\end{array}$ & Analyzed & $\begin{array}{l}\text { HCO } \\
\text { found }\end{array}$ & $\begin{array}{l}\text { High colo- } \\
\text { ny counts² }\end{array}$ & \\
\hline $\begin{array}{l}\text { Olympus } \\
{[77,126]}\end{array}$ & 1736 & 1583 & 1369 & $\begin{array}{l}74 \\
(5.4 \%)\end{array}$ & $6(0.4 \%)$ & 1932 & 1488 & $\begin{array}{l}75 \\
(5.0 \%)\end{array}$ & $9(0.6 \%)$ & $\begin{array}{l}444 \\
(23.0 \%)\end{array}$ \\
\hline $\begin{array}{l}\text { Pentax } \\
{[78,127]}\end{array}$ & 850 & 505 & 505 & $\begin{array}{l}40 \\
(7.9 \%)\end{array}$ & $18(3.6 \%)$ & $\begin{array}{l}\text { Data not } \\
\text { reported }\end{array}$ & 653 & $\begin{array}{l}32 \\
(4.9 \%)\end{array}$ & $29(4.4 \%)$ & $\begin{array}{l}98 \\
(13.0 \%)\end{array}$ \\
\hline $\begin{array}{l}\text { Fujifilm } \\
{[79,80]}\end{array}$ & 727 & 104 & 104 & $\begin{array}{l}2 \\
(1.9 \%)\end{array}$ & $1(1.0 \%)$ & $\begin{array}{l}\text { Data not } \\
\text { reported }\end{array}$ & $\begin{array}{l}\text { Data not } \\
\text { reported }^{3}\end{array}$ & $\begin{array}{l}\text { Data not } \\
\text { reported }^{3}\end{array}$ & $\begin{array}{l}\text { Data not } \\
\text { reported }\end{array}$ & 0 \\
\hline \multicolumn{11}{|c|}{$\begin{array}{l}\text { HCO: high-concern organisms } \\
1 \text { The number of discarded samples that contained } 1-10 \text { CFU or } 11-99 \text { CFU of low- or moderate-concern organisms was not specified } \\
2 \text { Includes cases where there were }>100 \text { CFU of low- or moderate-concern organisms } \\
{ }^{3} \text { The final report stated "Fujifilm has not enrolled a sufficient number of sites or collected a sufficient number of samples to establish a real-world contamination } \\
\text { rate." Data previously reported in the database appears to have been redacted. }\end{array}$} \\
\hline
\end{tabular}


or moderate-concern organisms. The presence of $>10$ colonies is considered actionable by CDC and Australian guidelines [85, $86]$, and other international guidelines recommend a benchmark of 20 CFU $[87,88]$ ( $\$$ Table 5 ). The exclusion of hundreds of samples raises questions about whether those samples had substantial bioburden and if microbes found were due to sampling errors. Despite these limitations, these data confirm that reprocessing does not reliably eliminate contamination.

\section{Clinical implications of underestimating infection risks}

\section{Neglecting to notify or test exposed patients}

In addition to delayed recognition and reporting of infections, a lack of transparency and poor inter-agency communication erode the ability of clinicians and infection preventionists to accurately assess infection risk and develop strategies to address breaches and patient exposure. In one case, hospital personnel observed blood on a patient-ready EUS endoscope, and investigators determined that four patients had been exposed to blood and bodily fluids because improper irrigation system connectors were used during cleaning and disinfection. Further investigation revealed that incorrect channel connectors had been used for 3 years, resulting in a lack of cleaning and HLD that placed numerous patients at risk [89]. The hospital notified 2,557 exposed patients, but did not recommend follow-up testing because the CDC and other experts advised that "the risk of transmission of any disease to patients is very remote" [13]. The lack of follow-up testing prevented characterization of actual infection risk.

\section{Public health risks}

Colonized or infected patients may serve as carriers, and secondary transmission has been documented in at least five outbreaks ( $>$ Table 1 ). Following an outbreak of NDMEscherichia coli in Chicago, 19 of 31 infected ERCP patients were eventually admitted to other hospitals for continuing care [90]. Ray et al. subsequently documented transmission of the superbug to 10 patients in six hospitals [90]. The risk of direct exposure and secondary transmission is heightened by a failure to adequately identify and report infections to stakeholders in local communities and beyond. Currently, there is no suitable reporting or notification system.

The impact of antimicrobial therapy on the risk of infection and superbug development is a major concern. The CDC and WHO have prioritized implementation of antimicrobial stewardship programs [91,92]. Several institutions evaluated their use of prophylactic antimicrobials and found no significant effect on infectious complication rates $[93,94]$. Du et al. noted that antibiotic prophylaxis guidelines do not consider patients' resistance profiles, and $62 \%$ to $73 \%$ were resistant to recommended antibiotics [95]. They attributed high rates of antimicrobial resistance to excessive antibiotic use [95]. Masadeh et al. observed that patients who received post-ERCP antibiotics were more likely to have resistant microbes [93]. Wendorf et al. hypothesized that antibiotics given to outbreak patients drove the development of additional resistance in the outbreak strain [96].

\section{Evidence-based calculations of infection risk}

Estimates of pathogen transmission with HCOs can be made using duodenoscope contamination rates that range from $0.3 \%$ in academic centers with rigorous adherence to reprocessing guidelines and duodenoscope maintenance [34,42] to $5 \%$ in FDA post-market surveillance studies conducted in 26 US facilities [76-78,80], 22\% in 67 Dutch hospitals [24], and $60 \%$ in other high-volume settings [37]. With 750,000 estimated annual ERCP procedures, this means that 2,250 or 37,500 , or even 412,500 procedures are performed with contaminated duodenoscopes annually in the United States. Using an average attack rate documented in settings where contaminated duodenoscopes were used (18.9\% [132/699]; > Table 1), this translates into a per-procedure HCO transmission rate of 1 in 1,765 ( $0.3 \%$ contaminated), 1 in 106 (5\% contaminated), 1 in 24 ( $22 \%$ contaminated), or 1 in 10 ERCP procedures (60\% contaminated). These calculated transmission rates reflect the full spectrum of disease with most patients developing long-standing asymptomatic colonization and only a minority manifesting more severe forms of PB, urinary tract, pulmonary, or vascular infections.

\section{Reducing the risk of ERCP-associated infections}

Reducing the risk of ERCP-associated infection will require a multifaceted approach including:

1. Prioritizing the improvement of reprocessing effectiveness by:

a) Establishing educational programs that support realworld competencies (e. g., hands-on and train-the-trainer programs; simulators)

b) Providing rigorous training and oversight to ensure adherence to optimal practices

c) Advocating for automation of manual cleaning and drying to reduce human error

d) Implementing the full range of quality assurance steps to ensure reprocessing effectiveness (e.g., leak tests, visual inspection, cleaning verification tests, HLD and sterilization monitoring, and drying verification)

2. Implementing mandatory duodenoscope servicing by:

a) Establishing an evidence-based schedule for routine inspections by biomedical department personnel or qualified repair technicians

b) Addressing defects that could injure patients or predispose endoscopes to harbor soil and microbial contamination

3. Enhancing the evidence base for assessing risks associated with ERCP by:

a) Conducting studies to evaluate real-world outcomes

b) Publishing findings from research and investigations that identify risk factors

c) Including sufficient information when reporting outbreaks, infections, or breaches (e. g., types of endo- 
scopes; number of patients exposed, tested, and infected or colonized; reprocessing methods and breaches; and maintenance issues or damage)

d) Evaluating antibiotic usage and its impact on transmission and resistance

e) Sharing innovations that may improve reprocessing effectiveness and patient safety

4. Partnering with manufacturers and biomedical engineers to address risks by:

a) Considering alternatives to conventional reusable devices (e.g., duodenoscopes that are sterilizable, single-use, or have disposable components that facilitate reprocessing) b) Evaluating the impact of these innovations on outcomes

\section{Conclusions}

Until recently, many clinicians and researchers believed the risk of post-ERCP infection was extremely low. There is now substantial evidence that duodenoscope reprocessing does not reliably eliminate soil or bioburden, allowing potential pathogens to remain on endoscopes. This clearly causes infections that harm patients and jeopardize public health, with evidence suggesting infections could be expected to occur in as few as 1 in 1,765 or as many as $10 \%$ of ERCP procedures when contaminated duodenoscopes are used. Endoscopists can lead efforts in reducing risk by working with a multidisciplinary team that includes infection preventionists, reprocessing and endoscopy personnel, and biomedical engineers. This team should develop and implement evidence-based strategies to improve reprocessing practices and systemically evaluate and report patient outcomes.

\section{Acknowledgements}

Our work on this manuscript was supported in part by an unrestricted research grant from Boston Scientific Corporation (BSC). BSC was not involved in developing our research approach, compiling or analyzing data, interpreting results, or preparing the manuscript, and the company was not provided with a copy of this manuscript for review prior to submission.

\section{Competing interests}

Ofstead, Buro, Hopkins, Eiland and Wetzler have received research grants, study materials, educational materials, or consulting contracts from 3 M Company, Ambu, Auris Health, Advanced Sterilization Products, Boston Scientific Corporation, Cogentix, Convergascent, Endoscopy Repair Specialists, Fortive, Healthmark, Invendo Medical, Laborie, Medivators, Mobile Instrument, Nanosonics, and Steris. Dr. Lichtenstein reports personal fees from Olympus America Inc. and Boston Scientific Corporation outside of the submitted work; has received consulting or speaking contracts from Augmenix, Iterative Scopes, Aries Pharmaceutical, and GI Supply; and has served as a principal investigator and advisor for Iterative Scopes and Motus.

\section{References}

[1] Kimmery MB, Burnett DA, Carr-Locke DL et al. ASGE Technology Assessment position paper: transmission of infection by gastrointestinal endoscopy. Gastrointest Endosc 1993; 39: 885-888

[2] Rutala WA, Weber D], Healthcare Infection Control Practices Advisory Committee (HICPAC). Guideline for Disinfection and Sterilization in Healthcare Facilities, 2008. Department of Health and Human Services; 2008

[3] ASGE Standards of Practice Committee. Banerjee S, Shen B et al. Infection control during Gl endoscopy. Gastrointest Endosc 2008; 67: 781-790

[4] Petersen BT, Chennat J, Cohen J et al. Multisociety guideline on reprocessing flexible Gl endoscopes: 2011. Infect Cont Hosp Epidemiol 2011; 32: 527-537

[5] Society of Gastroenterology Nurses and Associates. SGNA Standards: standards of infection control in reprocessing of flexible gastrointestinal endoscopes. Gastroenterol Nurs 2010; 33: 70-80

[6] Spach DH, Silverstein FE, Stamm WE. Transmission of infection by gastrointestinal endoscopy and bronchoscopy. Ann Intern Med 1993; 118: 117-128

[7] Ofstead CL, Dirlam Langlay AM, Mueller NJ et al. Re-evaluating endoscopy-associated infection risk estimates and their implications. Am J Infect Control 2013; 41: 734-736

[8] Grein JD, Murthy RK. New developments in the prevention of gastrointestinal scope-related infections. Infect Dis Clin N Am 2018: doi:10.1016/j.idc.2018.06.008

[9] Muscarella LF. Use of ethylene-oxide gas sterilisation to terminate multidrug-resistant bacterial outbreaks linked to duodenoscopes. BMJ Open Gastroenterol 2019; 6: e000282

[10] Calderwood AH, Day LW, Muthusamy VR et al. ASGE guideline for infection control during $\mathrm{Gl}$ endoscopy. Gastrointest Endosc 2018; 87: 1167-1179

[11] Bails J. Candor praised in scope problems. Pittsburgh Tribune-Review 2005: Available from: http://www.pittsburghlive.com/x/pittsburghtrib/trib/tribeast/s_319469.html

[12] Young M. No increase in infections despite sterilization problem, officials say. Vancouver Sun 2010: Available from: http://www.vancouversun.com/increase+infections+despite+sterilization+problem +officials/3955640/story.html

[13] Hill C. Error in manufacturer's disinfecting instructions prompts patient notification. Minneapolis: Hennepin County Medical Center; 2010

[14] Thornhill G, David M. Endoscope-associated infections: a microbiologist's perspective on current technologies. Tech Gastrointest Endosc 2019: doi:10.1016/j.tgie.2019.150625

[15] Saleh N. Post-endoscopic infection rates are higher than '1 in a million'. MDLinx ed, In the News 2018: Available from: https://www. mdlinx.com/gastroenterology/article/2029

[16] Food and Drug Administration. Design of endoscopic retrograde cholangiopancreatography (ERCP) duodenoscopes may impede effective cleaning: FDA Safety Communication. 2015: Available from: http://www.fda.gov/MedicalDevices/Safety/AlertsandNotices/ ucm434871.htm

[17] Wang P, Xu T, Ngamruengphong $S$ et al. Rates of infection after colonoscopy and osophagogastroduodenoscopy in ambulatory surgery centres in the USA. Gut 2018; 0: 1-11

[18] Council for International Organizations of Medical Science. Definitions. Available from: https://www.who.int/medicines/areas/quality_safety/safety_efficacy/trainingcourses/definitions.pdf

[19] Loor MM, Morancy JD, Glover JK et al. Single-setting endoscopic retrograde cholangiopancreatography (ERCP) and cholecystectomy 
improve the rate of surgical site infection. Surg Endosc 2017; 31: 5135-5142

[20] Ofstead CL, Doyle EM, Eiland JE et al. Practical toolkit for monitoring endoscope reprocessing effectiveness: identification of viable bacteria on gastroscopes, colonoscopes, and bronchoscopes. Am J Infect Control 2016; 44: 815-819

[21] England D, Houseman J, Horn L et al. Documented transmission of extended-spectrum beta-lactamase-producing Klebsiella pneumoniae from patient to gastroscope. Infect Control Hosp Epidemiol 2016; 37: 493-494

[22] Carbonne A, Thiolet JM, Fournier S et al. Control of a multi-hospital outbreak of KPC-producing Klebsiella pneumoniae type 2 in France, September to October 2009. Euro Surveill 2010; 15: 1-6

[23] Kim S, Russell D, Mohamadnejad M et al. Risk factors associated with the transmission of carbapenem-resistant Enterobacteriaceae via contaminated duodenoscopes. Gastrointest Endosc 2016; 83: 1121-1129

[24] Rauwers AW, Voor In't Holt AF, Buijs JG et al. High prevalence rate of digestive tract bacteria in duodenoscopes: a nationwide study. Gut 2018: doi:10.1136/gutjnl-2017-315082

[25] Epstein L, Hunter JC, Arwady MA et al. New Delhi metallo-beta-lactamase-producing carbapenem-resistant Escherichia coli associated with exposure to duodenoscopes. JAMA 2014; 312: 1447-1455

[26] Frias M, Tsai V, Moulton-Meissner $\mathrm{H}$ et al. Notes from the field: New Delhi-metallo-beta-lactamase-producing Escherichia coli associated with endoscopic retrograde cholangiopancreatography - Illinois, 2013. Morb Mortal Wkly Rep 2014; 62: 1051-1051

[27] Food and Drug Administration. Olympus Medical Systems Corp. Evis Exera Duodenovideoscope. MAUDE Adverse Event Report 8379810 Silver Spring, MD: 2019

[28] Food and Drug Administration. Olympus Medical Systems Corp. Evis Exera Duodenovideoscope. MAUDE Adverse Event Report 8204386 Silver Spring, MD: 2019

[29] Chandrasekhara V, Khashab MA, Muthusamy VR et al. Adverse events associated with ERCP. Gastrointest Endosc 2017; 85: 32-47

[30] Thosani N, Zubarik RS, Kochar R et al. Prospective evaluation of bacteremia rates and infectious complications among patients undergoing single-operator choledochoscopy during ERCP. Endoscopy 2016; 48: 424-431

[31] Bai Y, Gao F, Gao J et al. Prophylactic antibiotics cannot prevent endoscopic retrograde cholangiopancreatography-induced cholangitis: a meta-analysis. Pancreas 2009; 38: 126-130

[32] Khashab MA, Chithadi KV, Acosta RD et al. Antibiotic prophylaxis for Gl endoscopy. Gastrointest Endosc 2015; 81: 81-89

[33] Kullman E, Borch K, Lindström E et al. Bacteremia following diagnostic and therapeutic ERCP. Gastrointest Endosc 1992; 38: 444449

[34] Bartles RL, Leggett JE, Hove S et al. A randomized trial of single versus double high-level disinfection of duodenoscopes and linear echoendoscopes using standard automated reprocessing. Gastrointest Endosc 2018; 88: 306-313

[35] Rex DK, Sieber M, Lehman GA et al. A double-reprocessing high-leve disinfection protocol does not eliminate positive cultures from the elevators of duodenoscopes. Endoscopy 2017; 50: 588-596

[36] Snyder GM, Wright SB, Smithey A et al. Randomized comparison of 3 high-level disinfection and sterilization procedures for duodenoscopes. Gastroenterology 2017; 153: 1018-1025

[37] Visrodia K, Hanada Y, Pennington KM et al. Duodenoscope reprocessing surveillance with adenosine triphosphate testing and terminal cultures: a clinical pilot study. Gastrointest Endosc 2017; 86: $180-186$
[38] Gromski M, Sieber M, Sherman S et al. Double high-level disinfection vs. sterilization for reprocessing of duodenoscopes used for ERCP: a prospective study. Am J Gastroenterol 2019; 114: S1

[39] Verfaillie C], Bruno MJ, Voor in't holt AF et al. Withdrawal of a noveldesign duodenoscope ends outbreak of a VIM-2-producing Pseudomonas aeruginosa. Endoscopy 2015; 47: 493-502

[40] Alrabaa SF, Nguyen P, Sanderson R et al. Early identification and control of carbapenemase-producing Klebsiella pneumoniae, originating from contaminated endoscopic equipment. Am J Infect Control 2013; 41: 562-564

[41] Epstein L, Hunter J, Arwady A. Epi-Aid Trip Report: outbreak of New Delhi metallo-beta-lactamase-producing. E. coli at an acute care hospital, Chicago Region. Centers for Disease Control and Prevention Atlanta, GA: Department of Health and Human Services; 2013

[42] Ross AS, Baliga C, Verma P et al. A quarantine process for the resolution of duodenoscope-associated transmission of multidrug-resistant Escherichia coli. Gastrointest Endosc 2015; 82: 477-483

[43] Rauwers AW, Troelstra A, Fluit AC et al. Independent root cause analysis of contributing factors, including dismantling of 2 duodenoscopes, to an outbreak of multidrug-resistant Klebsiella pneumoniae. Gastrointest Endosc 2019: doi:10.1016/j.gie.2019.05.016

[44] Aumeran C, Poincloux L, Souweine B et al. Multidrug-resistant Klebsiella pneumoniae outbreak after endoscopic retrograde cholangiopancreatography. Endoscopy 2010; 42: 895-899

[45] Food and Drug Administration. Supplemental measures to enhance duodenoscope reprocessing: FDA Safety Communication. 2015: Available from: https://www.fdanews.com/ext/resources/files/0815/081015-duodenoscopes-fda.pdf?1520541508

[46] Dylla B, Kohner P, Ihde S et al. Comparison of two methods for culturing reprocessed duodenoscopes. Boston, MA: ASM Microbe; 2016

[47] Ofstead CL, Heymann OL, Quick MR et al. The effectiveness of sterilization for flexible ureteroscopes: a real-world study. Am J Infect Control 2017; 45: 888-895

[48] Chapman CG, Siddiqui UD, Manzano M et al. Risk of infection transmission in curvilinear array echoendoscopes: results of a prospective reprocessing and culture registry. Gastrointest Endosc 2017; 85: 390-397.e391

[49] Saliou P, Le Bars H, Payan C et al. Measures to improve microbial quality surveillance of gastrointestinal endoscopes. Endoscopy 2016; 48: 704-710

[50] Legemate JD, Kamphuis GM, Freund JE et al. Pre-use ureteroscope contamination after high-level disinfection: reprocessing effectiveness and the relation with cumulative ureteroscope use. J Urol 2019 doi:10.1097/ju.0000000000000108

[51] Ofstead CL, Wetzler HP, Eiland JE et al. Assessing residual contamination and damage inside flexible endoscopes over time. Am J Infect Control 2016; 44: 1675-1677

[52] Ofstead CL, Quick MR, Wetzler HP et al. Effectiveness of reprocessing for flexible bronchoscopes and endobronchial ultrasound bronchoscopes. Chest 2018; 154: 1024-1034

[53] Ofstead CL, Wetzler HP, Heymann OL et al. Longitudinal assessment of reprocessing effectiveness for colonoscopes and gastroscopes: results of visual inspections, biochemical markers, and microbial cultures. Am J Infect Control 2017; 45: e26-e33

[54] Ofstead CL, Wetzler HP, Doyle EM et al. Persistent contamination on colonoscopes and gastroscopes detected by biologic cultures and rapid indicators despite reprocessing performed in accordance with guidelines. Am J Infect Control 2015; 43: 794-801

[55] Ofstead CL, Heymann OL, Quick MR et al. Residual moisture and waterborne pathogens inside flexible endoscopes: evidence from a multisite study of endoscope drying effectiveness. Am J Infect Control 2018; 46: 689-696 
[56] Ofstead CL, Wetzler HP, Snyder AK et al. Endoscope reprocessing methods: a prospective study on the impact of human factors and automation. Gastroenterol Nurs 2010; 33: 304-311

[57] Food and Drug Administration. The FDA provides interim results of duodenoscope reprocessing studies conducted in real-world settings: FDA Safety Communication. 2018: Available from: https:// www.fda.gov/medical-devices/safety-communications/fda-provides-interim-results-duodenoscope-reprocessing-studies-conducted-real-world-settings-fda

[58] The Joint Commission. 4-1-1 on survey enhancements: new scoring revisions of IC.02.02.01. 2018: Available from: https://www.jointcommission.org/issues/article.aspx?Article =G/xUdh/VKujx]12FY7q2iOousSBZsyucrGSdk+Hj108=

[59] Armellino D, Cifu K, Wallace M et al. Implementation of remote video auditing with feedback and compliance for manual-cleaning protocols of endoscopic retrograde cholangiopancreatography endoscopes. Am J Infect Control 2018; 46: 594-596

[60] Thaker AM, Kim S, Sedarat A et al. Inspection of endoscope instrument channels after reprocessing using a prototype borescope. Gastrointest Endosc 2018; 88: 612-619

[61] Barakat MT, Girotra M, Huang RJ et al. Scoping the scope: endoscopic evaluation of endoscope working channels with a new highresolution inspection endoscope (with video). Gastrointest Endosc 2018; 88: 601-611

[62] The Joint Commission. The 5 most challenging requirements for first half of 2018. 2018: Available from: https://www.jointcommission. org/sitecore/media-library/tjc/newsletters/jc_online_sept_192pdf/

[63] Kumarage J, Khonyongwa K, Khan A et al. Transmission of MDR Pseudomonas aeruginosa between two flexible ureteroscopes and an outbreak of urinary tract infection: the fragility of endoscope decontamination. J Hosp Infect 2019; 102: 89-94

[64] Food and Drug Administration. Medivators Advantage Plus Automated Endoscope Reprocessor. MAUDE Adverse Event Report 8287300 Silver Spring, MD: 2019

[65] Food and Drug Administration. Medivators DSD-201 Automated Endoscope Reprocessor. MAUDE Adverse Event Report 8524712 Silver Spring, MD: 2019

[66] Barakat MT, Huang RJ, Banerjee S. Comparison of automated and manual drying in the elimination of residual endoscope working channel fluid after reprocessing (with video). Gastrointest Endosc 2019; 89: 124-132e122

[67] Galdys AL, Marsh JW, Delgado E et al. Bronchoscope-associated clusters of multidrug-resistant Pseudomonas aeruginosa and carbapenem-resistant Klebsiella pneumoniae. Infect Control Hosp Epidemiol 2019; 40: 40-46

[68] Foshay K. Medical scope now tied to Wisconsin superbug outbreak. 2015: Available from: http://america.aljazeera.com/watch/shows/ america-tonight/articles/2015/3/4/Medical-scope-tied-to-Wisconsin-superbug-outbreak.html

[69] Food and Drug Administration. Olympus Medical System Corporation Evis Exera II Duodenovideoscope. MAUDE Adverse Event Report 3608977 Silver Spring, MD: 2014

[70] Food and Drug Administration. Olympus Medical System Corporation Evis Exera II Duodenovideoscope. MAUDE Adverse Event Report 3608996 Silver Spring, MD: 2014

[71] Food and Drug Administration. Olympus Medical System Corporation Evis Exera II Duodenovideoscope. MAUDE Adverse Event Report 3608981 Silver Spring, MD: 2014

[72] Smith ZL, Oh YS, Saeian K et al. Transmission of carbapenem-resistant Enterobacteriaceae during ERCP: time to revisit the current reprocessing guidelines. Gastrointest Endosc 2015; 81: 1041-1045

[73] Humphries RM, Yang S, Kim S et al. Duodenoscope-related outbreak of a carbapenem-resistant Klebsiella pneumoniae identified using advanced molecular diagnostics. Clin Infect Dis 2017; 65: 11591166

[74] Jousset AB, Bonnin RA, Rosinski-Chupin I et al. 4.5 years within-patient evolution of a colistin resistant KPC-producing Klebsiella pneumoniae ST258. Clin Infect Dis 2018: doi:10.1093/cid/ciy293

[75] Food and Drug Administration. FDA orders duodenoscope manufacturers to conduct postmarket surveillance studies in health care facilities. 2015: Available from: http://www.fda.gov/NewsEvents/ Newsroom/PressAnnouncements/ucm465639.htm

[76] Food and Drug Administration. Statement from Jeff Shuren, M.D., Director of the Center for Devices and Radiological Health, on continued efforts to assess duodenoscope contamination risk. 2019: Available from: https://www.fda.gov/news-events/press-announcements/statement-jeff-shuren-md-jd-director-center-devices-andradiological-health-updated-safety

[77] Food and Drug Administration. Olympus 522 Post Market Surveillance Studies-Sampling and culturing study. Department of Health and Human Services. 2020: Available from: https://www.accessdata. fda.gov/scripts/cdrh/cfdocs/cfPMA/pss.cfm?t_id=354\&_id=3726

[78] Food and Drug Administration. Pentax 522 Post Market Surveillance Studies-Sampling and culturing study. Department of Health and Human Services. 2020: Available from: https://www.accessdata.fda. gov/scripts/cdrh/cfdocs/cfPMA/pss.cfm?t_id=355\&__id=3727

[79] Food and Drug Administration. Fujifilm 522 Postmarket Surviellance Studies-Sampling and culturing study. Department of Health \& Human Services. 2018: Available from: https://www.accessdata.fda. gov/scripts/cdrh/cfdocs/cfPMA/pss.cfm?t_id=353\&__id=3725

[80] Food and Drug Administration. Fujifilm 522 Post Market Surveillance Studies-Sampling and culturing study. Department of Health and Human Services. 2020: Available from: https://www.accessdata.fda. gov/scripts/cdrh/cfdocs/cfPMA/pss.cfm?t_id=353\&__id=3725

[81] Alfa MJ, Singh H. Impact of wet storage and other factors on biofilm formation and contamination of patient-ready endoscopes: a narrative review. Gastrointest Endosc 2019: doi:10.1016/j. gie.2019.08.043

[82] Food and Drug Administration. Olympus Medical Systems Corp. Evis Exera II Duodenovideoscope. MAUDE Adverse Event Report 8030512 Silver Spring, MD: 2018

[83] Food and Drug Administration. Olympus Medical Systems Corp. Evis Exera II Duodenovideoscope. MAUDE Adverse Event Report 8034924 Silver Spring, MD: 2018

[84] Food and Drug Administration. Olympus Medical Systems Corp. Evis Exera II Duodenovideoscope. MAUDE Adverse Event Report 8365270 Silver Spring, MD: 2019

[85] Centers for Disease Control and Prevention. Interim protocol for healthcare facilities regarding surveillance for bacterial contamination of duodenoscopes after reprocessing. Department of Health \& Human Services; 2015

[86] Taylor A, Jones D, Everts R et al. Infection Control in Endoscopy. 3rd ed. Gastroenterological Society of Australia Gastroenterological Nurses College of Australia; 2011

[87] Beilenhoff U, Neumann CS, Rey JF et al. ESGE-ESGENA guideline for quality assurance in reprocessing: microbiological surveillance testing in endoscopy. Endoscopy 2007; 39: 175-181

[88] Public Health Agency of Canada. Infection prevention and control guideline for flexible gastrointestinal endoscopy and flexible bronchoscopy. Infection Prevention and Control Program 2010

[89] Minnesota Department of Health. Hennepin County Medical Center investigative report and CMS-2567. Office of Health Facility Complaints; 2010

[90] Ray M], Lin MY, Tang AS et al. Regional spread of an outbreak of carbapenem-resistant Enterobacteriaceae (CRE) through an ego network of healthcare facilities. Clin Infect Dis 2018: doi:10.1093/ cid/ciy084 
[91] World Health Organization. Global action plan on antimicrobial resistance. Geneva, Switzerland: 2015

[92] Centers for Disease Control and Prevention. Core elements of antibiotic stewardship. Antibiotic Prescribing and Use in Hospitals and Long-Term Care 2019: Available from: https://www.cdc.gov/antibiotic-use/core-elements/index.html

[93] Masadeh M, Chandra S, Livorsi D et al. Evaluation of biliary bacterial resistance in patients with frequent biliary instrumentation, one size does not fit all. Dig Dis Sci 2018: doi:10.1007/s10620-018-5263-5

[94] Ishigaki T, Sasaki T, Serikawa M et al. Evaluation of antibiotic use to prevent post-endoscopic retrograde cholangiopancreatography pancreatitis and cholangitis. Hepatogastroenterology 2015; 62: 417-424

[95] Du M, Suo J, Liu B et al. Post-ERCP infection and its epidemiological and clinical characteristics in a large Chinese tertiary hospital: a 4year surveillance study. Antimicrob Resist Infect Control 2017; 6: 131

[96] Wendorf KA, Kay M, Baliga C et al. Endoscopic retrograde cholangiopancreatography-associated AmpC Escherichia coli outbreak. Infect Control Hosp Epidemiol 2015; 36: 634-642

[97] Bourigault C, Le Gallou F, Bodet N et al. Duodenoscopy: an amplifier of cross-transmission during a carbapenemase-producing Enterobacteriaceae outbreak in a gastroenterology pathway. J Hosp Infect 2018: doi:10.1016/j.jhin.2018.04.015

[98] Shenoy ES, Pierce VM, Walters MS et al. Transmission of mobile colistin resistance (mcr-1) by duodenoscope. Clin Infect Dis 2018; 68: $1327-1334$

[99] Robertson P, Smith A, Anderson M et al. Transmission of Salmonella enteritidis after endoscopic retrograde cholangiopancreatography because of inadequate endoscope decontamination. Am J Infect Control 2017; 45: 440-442

[100] Kola A, Piening B, Pape UF et al. An outbreak of carbapenem-resistant OXA-48 - producing Klebsiella pneumonia associated to duodenoscopy. Antimicrob Resist Infect Control 2015; 4: 8

[101] Marsh JW, Krauland MG, Nelson JS et al. Genomic epidemiology of an endoscope-associated outbreak of Klebsiella pneumoniae carbapenemase (KPC)-producing K. pneumoniae. PloS One 2015; 10: e0144310

[102] Qiu L, Zhou Z, Liu Q et al. Investigating the failure of repeated standard cleaning and disinfection of a Pseudomonas aeruginosa-infected pancreatic and biliary endoscope. Am J Infect Control 2015; 43: e $43-46$

[103] Sanderson R, Braithwaite L, Ball L et al. An outbreak of carbapenemresistant Klebsiella pneumoniae infections associated with endoscopic retrograde cholangiopancreatography (ERCP) procedures at a hospital. Am J Infect Control 2010; 38: e141

[104] Sanderson R. An outbreak of carbapenem-resistant Klebsiella pneumoniae infections associated with endoscopic retrograde cholangiopancreatography (ERCP) procedures at a hospital. APIC. New Orleans, LA: 2010

[105] Centers for Medicare and Medicaid Services. Advocate Lutheran General Hospital - CMS-2567 Statement of Deficiencies and Plan of Correction. Department of Health and Human Services; 2014

[106] Yang S, Hemarajata P, Hindler J et al. Evolution and transmission of carbapenem-resistant Klebsiella pneumoniae expressing the blaOXA232 gene during an institutional outbreak associated with endoscopic retrograde cholangiopancreatography. Clin Infect Dis 2017; 64: 894-901

[107] University of California - Los Angeles. UCLA statement on notification of patients regarding endoscopic procedures. 2015: Available from: https://www.uclahealth.org/body.cfm?id=1397\&action=detail\&ref=1216\&fr=true
[108] Rubin Z. Carbapenem resistant Klebsiella pneumoniae following endoscopic retrograde cholangiopancreatography at Ronald Reagan University Medical Center. FDA Gastroenterology-Urology Devices Panel. Washington, D.C: UCLA Health System; 2015

[109] Centers for Medicare and Medicaid Services. Ronald Reagan UCLA Medical Center Statement of Deficiencies and Plan of Correction. 2015: 1-42

[110] Hunter J, Epstein L. Epi-Aid Trip Report: cluster of plasmid-mediated AmpC-producing carbapenem-resistant Enterobacteriaceae (CRE) Washington, 2014 (Epi-2014-043). Atlanta, GA: Centers for Disease Control and Prevention; 2014: 24

[111] Food and Drug Administration. Olympus Medical System Corporation Olympus Duodenovideoscope. MAUDE Adverse Event Report 4077782 Silver Spring, MD: 2014

[112] Food and Drug Administration. Olympus Medical System Corporation Olympus Duodenovideoscope. MAUDE Adverse Event Report 4083574 Silver Spring, MD: 2014

[113] Centers for Medicare and Medicaid Services. Virginia Mason Medical Center - Case file. 2015

[114] Food and Drug Administration. Olympus Medical Systems Corp. MAUDE Adverse Event Report 8177954 Silver Spring, MD: 2019

[115] Food and Drug Administration. Olympus Medical Systems Corp. Evis Exera Duodenovideoscope. MAUDE Adverse Event Report 8820754 Silver Spring, MD: 2019

[116] Food and Drug Administration. Olympus Medical Systems Corp. Evis Lucera Duodenovideoscope. MAUDE Adverse Event Report 8538532 Silver Spring, MD: 2019

[117] Food and Drug Administration. Olympus Medical Systems Corp. Evis Exera II Duodenovideoscope. MAUDE Adverse Event Report 7027139 Silver Spring, MD: 2017

[118] Food and Drug Administration. Olympus Medical Systems Corp. Evis Exera II Duodenovideoscope. MAUDE Adverse Event Report 8201861 Silver Spring, MD: 2018

[119] Food and Drug Administration. Olympus Medical Systems Corp. Evis Exera II Duodenovideoscope. MAUDE Adverse Event Report 8201871 Silver Spring, MD: 2018

[120] Food and Drug Administration. Pentax of America, Inc. Video Duodenoscope Duodenoscope and Accessories, Flexible/Rigid. MAUDE Adverse Event Report 7548459 Silver Spring, MD: 2018

[121] Food and Drug Administration. Olympus Medical Systems Corp. Evis Exera II Duodenovideoscope. MAUDE Adverse Event Report 8730284 Silver Spring, MD: 2019

[122] Food and Drug Administration. Olympus Medical Systems Corp. Evis Exera II Duodenovideoscope. MAUDE Adverse Event Report 8825520 Silver Spring, MD: 2019

[123] Food and Drug Administration. Olympus Medical Systems Corp. Evis Exera Duodenovideoscope. MAUDE Adverse Event Report 8751568 Silver Spring, MD: 2019

[124] Food and Drug Administration. Olympus Medical Systems Corp. Evis Exera II Duodenovideoscope. MAUDE Adverse Event Report 7791919 Silver Spring, MD: 2018

[125] Food and Drug Administration. Olympus Medical Systems Corp. Evis Exera II Duodenovideoscope. MAUDE Adverse Event Report 7424492 Silver Spring, MD: 2018

[126] Food and Drug Administration. Olympus 522 postmarket surveillance studies-Sampling and culturing study. Department of Health \& Human Services; 2019: Available from: https://www.accessdata. fda.gov/scripts/cdrh/cfdocs/cfPMA/pss.cfm?t_id=354\&__id=3726

[127] Food and Drug Administration. Pentax 522 Post Market Surveillance Studies-Sampling and culturing study. Department of Health \& Human Services; 2018: Available from: https://www.accessdata.fda. gov/scripts/cdrh/cfdocs/cfPMA/pss.cfm?t_id=355\&__id=3727 